Document downloaded from:

http://hdl.handle.net/10251/56987

This paper must be cited as:

Gutierrez Campo, AM.; Brimont ., ACJ.; Herrera Llorente, J.; Aamer ., M.; Thomson, DJ.; Gardes, FY.; Reed, GT... (2013). Analytical model for calculating the nonlinear distortion in silicon-based electro-optic Mach-Zehnder modulators. Journal of Lightwave Technology. 31(23):3603-3612. doi:10.1109/JLT.2013.2286838.

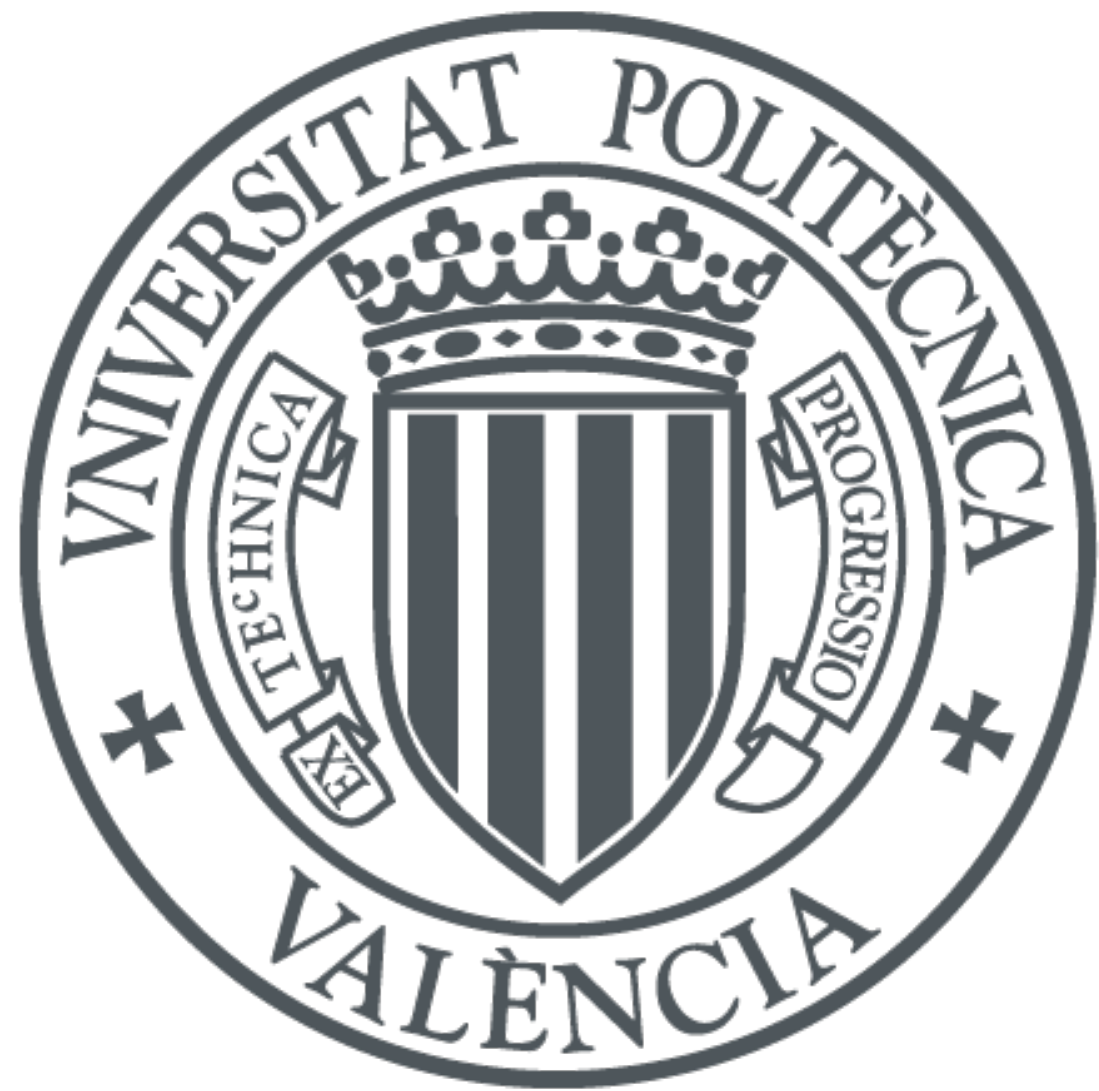

The final publication is available at

http://dx.doi.org/10.1109/JLT.2013.2286838

Copyright Institute of Electrical and Electronics Engineers (IEEE)

Additional Information 


\title{
Analytical Model for Calculating the Nonlinear Distortion in Silicon-Based Electro-Optic Mach-Zehnder Modulators
}

\author{
Ana M. Gutierrez, Antione Brimont, Javier Herrera, Mariam Aamer, Dave J. Thomson, Frederic \\ Y. Gardes, Graham T. Reed, Jean-Marc Fedeli and Pablo Sanchis, Member, IEEE
}

\begin{abstract}
In this work, an analytical model for calculating the nonlinear harmonic/intermodulation distortion for RF signals in silicon-based electro-optic modulators is investigated by considering the nonlinearity on the effective index change curve with the operation point and the device structure simultaneously. Distortion expressions are obtained and theoretical results are presented showing that optimal modulator parameters can be found to linearize it. Moreover, the harmonic distortion of a $1 \mathrm{~mm}$ silicon-based asymmetric MZI is RF characterized and used to corroborate the theoretical results. Based on the present model, the nonlinear distortion in terms of bias voltage or operating wavelength is calculated and validated by comparing with the experimental data, showing a good agreement between measurements and theory. Analog photonic link quality parameter like carrier-to-distortion is one of the parameters that can be found with that model. Finally, the modulation depth is measured to assure that no over-modulation is produced.
\end{abstract}

Index Terms-Silicon on insulator Technology, Integrated Optics, Nonlinear distortion, Electrooptic modulation.

\section{INTRODUCTION}

Y ear after year a gradual transition from electrical to optical technology is being carried out allowing much longer links with larger bandwidths. This transition arises from the increasing need to transfer the maximum amount of information with the least cost, increasing the complexity and requirements in the networks interconnects including on-chip and off-chip communications. Besides this gradual transition, recently, the distribution of analog radio frequency (RF) signals over the optical fibers is catching growing attention

Manuscript received January, 2013. This work was supported from TEC2012-38540 LEOMIS, TEC2008-06333 SINADEC and PROMETEO2010-087 R\&D Excellency Program (NANOMET).

A. M. Gutierrez, A. Brimont, A. Aamer and P. Sanchis are with the Nanophotonics Technology Center (NTC), Universitat Politecnica Valencia, 46022, Spain (e-mail: angucam@ntc.upv.es; abrimont@ntc.upv.es; maaa@ntc.upv.es; pabsanki@ntc.upv.es).

J. Herrera is with Fibernova Systems, S. L., Universitat Politecnica Valencia, 46022, Spain (e-mail: jherrera@fibernova.com)

D. J. Thomson, F. Y. Gardes and G. T. Reed are with the Optoelectronics Research Centre, University of Southampton, Southampton, UK (e-mail: d.thomson@soton.ac.uk,f.gardes@soton.ac.uk, g.t.reed@soton.ac.uk)

J. M. Fedeli is with CEA, LETI, Minatec Campus, 38054, Grenoble Cedex, France (e-mail: jean-marc.fedeli@cea.fr) and compounding the called analog photonics links (APL). These links, in which an RF signal is converted to the optical domain, distributed via optical fiber and reconverted to electrical format, are the heart of the profitable field of microwave photonics. This technology encompasses numerous potentially rewarding applications such as radio-over-fiber (RoF), antenna remoting, subcarrier transmission, signal processing or photonic analog-to-digital conversion [1, 2].

On the other hand, on-chip integration of discrete photonic components is a longstanding goal of integrated optics. Silicon-on-insulator (SOI) technology, that utilizes scalable CMOS technology, has turned out to be a suitable platform to reach impressive levels of integration of most high performance silicon photonics devices [3, 4]. So, the use of silicon photonic devices to generate and process analog RF signals may lead to chip-size reduction and power efficiency improvements. Among the numerous building blocks required to enable a fully functional photonic integrated chip along with high speed electronics, electro-optic modulators, which link the electrical and optical domain, are essential [5]. These are just the devices employed in APLs for converting the RF signals into the optical format and they are of paramount importance for achieving high performance links. The leading approach for developing electro-optical modulators in silicon photonics is currently via the plasma dispersion effect which consists of varying the free-carrier concentration in a doped silicon waveguide [6, 7]. The electro-optic mechanism achieved with the plasma dispersion is an inherent nonlinear effect. Moreover the cosine-squared Mach-Zehnder Interferometer (MZI) structure also shows a nonlinear transfer function. Therefore, the nonlinearity at the output of a siliconbased MZI modulator is originated basically from the operating bias point of the modulator nonlinear transfer function combined with the intrinsic nonlinear response of the material in the active area. These nonlinearities in the modulator create distortions which limit the performance of an APL, but also that can be exploited, for example using the electro-optic modulator as a photonic microwave mixer.

Significant milestones have been achieved during recent years through the plasma dispersion effect although mostly for digital applications [8-20], but so far very little attention has been paid to analog applications. As mentioned above, high 
linearity is required for RF signal transmission. In this case, the distortion terms closest to the frequency of the transmitted signal have to be reduced. A lot of methods to improve the commercial $\mathrm{LiNbO}_{3}$ modulators linearity have been published, using linearization schemes based on pre- and postcompensation distortion [21-23]. In silicon, a modulator linearization scheme was reported in [24] showing a siliconbased modulator with superior linearity with respect to a conventional MZI modulator. Moreover, other silicon-based modulators have been used for RoF transmission allowing very high features such 50dB [25] or 57dB [26] carrier-to-distortion ratio, showing significant advantages for emerging RoF applications. In contrast with the plain analog signal transport, many nonlinear applications such as mixing or frequency conversion are proposed techniques in the microwave photonics field. In this case, the nonlinear operation of the modulator is exploited, taking advantage of the distortion produced by the nonlinearities. The objective in that case is to maximize the farthest distortion terms. Many works have been reported about these techniques [27-31] using commercial $\mathrm{LiNbO}_{3}$ modulators. In silicon, we recently reported a photonic mixer with excellent quality parameters, such a $7 \mathrm{~dB}$ conversion losses and an error vector magnitude of $8 \%$, for frequency conversion applications [32].

Thus, the investigation of the distortion properties of silicon modulators is an important question for future all silicon analog photonic applications. In [24], the modulator linearity is analyzed theoretically, using models and numerical simulations. However, the expressions presented are limited to a symmetric push-pull configuration which automatically cancels the second order and higher-order even terms of the phase response. Moreover, in [25], the measurements are verified by simulation results showing the same trend, but no analytical expressions are presented. In this work, we develop an analytic model for calculating the distortion of a siliconbased electro-optic MZI modulator by taking into account the nonlinearity on the effective index change with the bias operation point and the device's structure simultaneously. The modulator transfer function is expanded into a Taylor series obtaining the second $\left(2^{\text {nd }}\right)$ and third $\left(3^{\text {rd }}\right)$ order harmonic and intermodulation terms so the model allows the designer to set a proper choice of modulator parameters to linearize the device or to enhance the nonlinearities. Finally, the harmonic distortions are validated with experimental measurements of a fabricated silicon MZI modulator.

\section{ANALYTICAL MODEL}

The plasma dispersion effect, the most commonly adopted electro-optic effect in silicon, consists of a change in the silicon refractive index by varying electrons and holes distribution within the material. The induced real refractive index $(\Delta \mathrm{n})$ produced by the free-carrier plasma dispersion effect at the wavelength of $1.55 \mu \mathrm{m}$ is calculated as follows [7]:

$$
\Delta n=\Delta \mathrm{n}_{\mathrm{e}}+\Delta \mathrm{n}_{\mathrm{h}}=-\left[8.8 \times 10^{-22} \Delta \mathrm{N}+8.5 \times 10^{-18} \Delta \mathrm{P}^{0.8}\right]
$$

where $\Delta \mathrm{n}_{\mathrm{e}}$ and $\Delta \mathrm{n}_{\mathrm{h}}$ are the refractive index changes due to electrons and holes concentration changes $\Delta \mathrm{N}$ and $\Delta \mathrm{P}$ (in $\mathrm{cm}^{-3}$ ). Equation (1) indicates that the index change by the plasma dispersion is a nonlinear effect [6, 7]. This nonlinear dependency produces intermodulation and harmonic distortion terms. In contrast with linear material-based modulators such $\mathrm{LiNbO}_{3}$, in which the distortion usually have the same performance with the bias point (at quadrature bias point, all even order distortion terms vanish, while odd order maximize), for a nonlinear material the distortion depends on several factors. So, this is the first motivation for the development of a model which can help to predict the distortion performance in a silicon-based modulator.

If the $\Delta \mathrm{n}$ index varies by plasma dispersion effect, there will be a change $\Delta \mathrm{n}_{\text {eff }}$ in effective index of the propagating mode. In general $\Delta \mathrm{n}$ can be regarded as proportional to $\Delta \mathrm{n}_{\text {eff. }}$. Therefore, the injection of an electric field causes a change in the effective refractive index of the guided mode, which in turn induces a change in the accumulated phase in the propagation distance $\mathrm{L}_{\mathrm{ACT}}$ :

$$
\Delta \Phi=\frac{2 \pi}{\lambda} \Delta n_{\text {eff }} L_{A C T}
$$

where $\Delta \mathrm{n}_{\text {eff }}$ is the effective index change, $\lambda$ is the wavelength and $L_{A C T}$ is the length of the active region. By embedding such optical phase modulation in a MZI structure, it is possible to convert the phase modulation into intensity modulation. The MZI transfer function between bias parameter, DC voltage or operating wavelength (in an asymmetric MZI structure), and optical output power is periodic with such bias parameter. This periodic function is a nonlinear cosine-squared, which also produces intermodulation and harmonic distortions.

Harmonic distortion can be defined as a single-tone distortion product caused by device nonlinearity. When a nonlinear device is stimulated by a signal at frequency $\mathrm{f}_{1}$, spurious output signals can be generated at the harmonic frequencies $2 f_{1}, 3 f_{1}, 4 f_{1}$, i.e. at $N f_{1}$. The order of the distortion product is given by the $\mathrm{N}$ frequency multiplier. Intermodulation distortion is a multi-tone distortion product that results when two or more signals are present at the input of a nonlinear device. The $2^{\text {nd }}$-order intermodulation products of two signals at $f_{1}$ and $f_{2}$ would occur at $f_{1}+f_{2}, f_{2}-f_{1}, 2 f_{1}$ and $2 f_{2}$ and $3^{\text {rd }}$-order at $2 f_{1}+f_{2}, 2 f_{1}-f_{2}, f_{1}+2 f_{2}$ and $f_{1}-2 f_{2}$, that is, at $\mathrm{Nf}_{1} \pm \mathrm{f}_{2}$ or $\mathrm{f}_{1} \pm \mathrm{Nf}_{2}$ with $\mathrm{N}=1$ for the $2^{\text {nd }}$-order terms and $\mathrm{N}=2$ for the $3^{\text {rd }}$ ones. Here and for the development of our model, we assume that the APL nonlinearity is dominated only by the modulation device. Thus, we assume that other devices in the APL, such us the photo-detector, are linear. These components also contribute to APL nonlinearities but generally their contributions are much smaller compared to that of the modulation devices [33] and, hence, most of the time can be neglected. 


\section{A. Model derivation}

The starting point of the model is the effective index change with the applied DC voltage. As we have already mentioned, this dependency is nonlinear for the silicon-based MZI. The nonlinear form of the effective index change can be approximated by a logarithmic curve [25, 34] as shown in (3):

$$
\Delta n_{\text {eff }}=k \ln \left(1+\frac{V_{a p p}}{V_{b}}\right)
$$

where $\mathrm{k}$ (dimensionless) and $\mathrm{V}_{\mathrm{b}}$ (in volts) are constants. In our case, we will use these parameters to fit the curve with the DC performance experimental results of the effective index variation. This effective index change induces a phase shift that accomplishes (2). Furthermore, in case of having a length difference between two arms of the MZI also induces an additional phase shift proportional to this length difference. Thereupon, we can regard that the total induced phase shift is due to two factors: the device structure, related with modulator transfer function, which has been called $\Delta \Phi_{\text {Bias }}$ and allows driving the modulator to the appropriate operation bias point; and the silicon in the active area, which has been named as $\Delta \Phi_{\mathrm{Si}}$, and it is described in (5). So, the final phase shift expression can be written as:

$$
\Delta \Phi_{\text {TOT }}=\Delta \Phi_{S i}+\Delta \Phi_{\text {Bias }}
$$

where:

$$
\Delta \Phi_{S i}=\frac{2 \pi}{\lambda} \Delta n_{e f f} L_{A C T}
$$

Finally, the detected optical power at the output of a MZI modulator is:

$$
P_{\text {out }}=P_{\text {in }} \cos ^{2}\left(\frac{\Delta \Phi_{T O T}}{2}\right)
$$

where $P_{\text {in }}$ is the input optical power to the modulator. If we substitute the total phase shift described by (4) in (6) and expand the cosine, we can state the output power as:

$$
P_{\text {out }}=P_{\text {in }}\left(\begin{array}{l}
\frac{1}{2}+\frac{1}{2} \cos \left(\Delta \Phi_{S i}\right) \cos \left(\Delta \Phi_{\text {Bias }}\right) \\
-\frac{1}{2} \sin \left(\Delta \Phi_{S i}\right) \sin \left(\Delta \Phi_{\text {Bias }}\right)
\end{array}\right)
$$

Also, the phase shift due to the silicon using the expressions (3) and (5) results:

$$
\Delta \Phi_{S i}=\frac{2 \pi}{\lambda} L_{A C T} \Delta n_{e f f}=\sigma \ln \left(1+\frac{V_{a p p}}{V_{b}}\right)
$$

where we have defined the design parameter $\sigma$ (in radians) as shown in (9):

$$
\sigma=\frac{2 \pi}{\lambda} L_{A C T} k
$$

On the other hand, in a two-tone test, the input signal takes the form:

$$
V_{r f}=V_{R F_{1}} \cos \left(\omega_{1} t\right)+V_{R F_{2}} \cos \left(\omega_{2} t\right)
$$

where $\mathrm{V}_{\mathrm{RF} 1}$ and $\mathrm{V}_{\mathrm{RF} 2}$ are the $\mathrm{RF}$ signals amplitudes and $\omega_{1}=2 \pi f_{1}$ and $\Phi_{2}=2 \pi f_{2}$ are the angular modulating frequencies. So, finally, the total applied voltage to the electro-optic modulator is the sum of one DC term and one RF term, as:

$$
V_{\text {app }}=V_{D C}+V_{r f}=V_{D C}+V_{R F_{1}} \cos \left(\omega_{1} t\right)+V_{R F_{2}} \cos \left(\omega_{2} t\right)
$$

So, if we make a compilation of the above equations and we substitute each equation into each other, we realize that the final expression for the silicon phase shift results:

$$
\Delta \Phi_{S i}=\sigma\left(Y_{D C}+\ln \left(1+X_{1} \cos \left(\omega_{1} t\right)+X_{2} \cos \left(\omega_{2} t\right)\right)\right)
$$

For simplicity, the model has been developed under the assumption that the two tone input signals are of equal amplitude and the small signal approximation $\mathrm{V}_{\mathrm{RF}}<<\mathrm{V}_{\mathrm{b}}$, so:

$$
X_{1}=X_{2}=X=\frac{V_{R F}}{V_{b}+V_{D C}}<<1
$$

Finally, we can write the silicon phase shift as described in (14):

$$
\Delta \Phi_{S i}=\sigma\left(Y_{D C}+\ln (1+Y)\right)
$$

where $\mathrm{Y}$ and $\mathrm{Y}_{\mathrm{DC}}$ are given by (15) and (16) respectively:

$$
\begin{gathered}
Y=X\left(\cos \left(\omega_{1} t\right)+\cos \left(\omega_{2} t\right)\right) \\
Y_{D C}=\ln \left(1+\frac{V_{D C}}{V_{b}}\right)
\end{gathered}
$$

Now, the silicon phase shift can be expanded into the natural logarithm Taylor series of $\ln (1+Y)$. The result will consist of a DC component which does not depend on $\Phi$, and a set of harmonics and intermodulation distortion terms. Finally, the silicon phase shift resulted of this Taylor series is:

$$
\Delta \Phi_{S i}=\sigma\left(\begin{array}{l}
-\frac{1}{2} X^{2}+\left(X+\frac{3}{4} X^{3}\right) \cos \left(\omega_{1} t\right) \\
-\frac{1}{4} X^{2} \cos \left(2 \omega_{1} t\right)+\frac{1}{12} X^{3} \cos \left(3 \omega_{1} t\right) \\
-\frac{1}{2} X^{2} \cos \left(\left(\omega_{1} \pm \omega_{2}\right) t\right)+\frac{1}{4} X^{3} \cos \left(\left(2 \omega_{1} \pm \omega_{2}\right) t\right)
\end{array}\right)
$$


Plugging this expression of the silicon phase shift into (7) and performing another Taylor expansion of the cosine and sine, the result is again a set of harmonic and intermodulation products. The amplitudes of all these components will be sums of linear combinations of $\mathrm{X}, \mathrm{Y}_{\mathrm{DC}}$ and $\sigma$. The desired expressions that describe the modulator amplitudes nonlinearities are finally obtained and described by (18) in which the sub-index ' $c$ ' is referring to cosine and ' $s$ ' to sine:

$$
\begin{aligned}
& P_{F U N D .}\left(\omega_{1}\right)=\left\{\begin{array}{l}
\frac{1}{2} \cos \left(\Delta \Phi_{\text {Bias }}\right)\left[\Phi_{F U N D C} X\right] \\
-\frac{1}{2} \sin \left(\Delta \Phi_{\text {Bias }}\right)\left[\Phi_{F U N D s} X\right]
\end{array}\right. \\
& P_{\text {IMD2 }}\left(\omega_{1} \pm \omega_{2}\right)=\left\{\begin{array}{l}
\frac{1}{2} \cos \left(\Delta \Phi_{\text {Bias }}\right)\left[\Phi_{I M D 2 c} X^{2}\right] \\
-\frac{1}{2} \sin \left(\Delta \Phi_{\text {Bias }}\right)\left[\Phi_{\text {IMD2s }} X^{2}\right]
\end{array}\right. \\
& P_{\text {IMD3 }}\left(2 \omega_{1} \pm \omega_{2}\right)=\left\{\begin{array}{l}
\frac{1}{2} \cos \left(\Delta \Phi_{\text {Bias }}\right)\left[\Phi_{\text {IMD3c }} X^{3}\right] \\
-\frac{1}{2} \sin \left(\Delta \Phi_{\text {Bias }}\right)\left[\Phi_{\text {IMD3s }} X^{3}\right]
\end{array}\right.
\end{aligned}
$$

As we can observe, the fundamental, $2^{\text {nd }}$-order and $3^{\text {rd }}$-order amplitudes are the terms with linear, quadratic and cubic dependence on the modulating signal $\mathrm{V}_{\mathrm{RF}}$, respectively. Finally, the cosine phase expressions are:

$$
\begin{aligned}
& \Phi_{F U N D C}=\left(-Y_{D C}\right) \sigma^{2} \\
& \Phi_{I M D 2 c}=\left(\frac{1}{2} Y_{D C}-\frac{1}{2}\right) \sigma^{2} \\
& \Phi_{I M D 3 c}=\left(-\frac{1}{2} Y_{D C}+\frac{3}{8}\right) \sigma^{2}
\end{aligned}
$$

And the sine phase expressions:

$$
\begin{aligned}
& \Phi_{F U N D S}=\sigma-\left(\frac{1}{2} Y_{D C}^{2}\right) \sigma^{3} \\
& \Phi_{I M D 2 s}=\left(-\frac{1}{2}\right) \sigma+\left(\frac{1}{4} Y_{D C}^{2}-\frac{1}{2} Y_{D C}\right) \sigma^{3} \\
& \Phi_{I M D 3 s}=\left(\frac{1}{4}\right) \sigma-\left(\frac{1}{8} Y_{D C}^{2}-\frac{3}{8} Y_{D C}+\frac{1}{8}\right) \sigma^{3}
\end{aligned}
$$

The IMD amplitudes differ from the HD amplitudes even though they are basically generated by the same mechanism. It has been found that the IMD2 amplitude is twice that of the HD2 amplitude while the IMD3 amplitude is three-times the HD3 amplitude. However, the powers of these components rather than the amplitudes are usually measured. If we regard the amplitude to be either a current or a voltage, then the power considered here is an electrical or an RF power. Thus we can easily identify that the powers of the distortion terms are proportional to the square of their amplitudes. So, we can deduce that the power of the HD2 terms expressed in decibels are approximately $6 \mathrm{~dB}$ lower compared to the IMD2 powers, and the HD3 powers are approximately $9.5 \mathrm{~dB}$ lower relative to the IMD3 powers. Therefore, we can affirm that:

$$
\begin{aligned}
& P_{H D 2}=P_{I M D 2}-6 d B \\
& P_{H D 3}=P_{I M D 3}-9.5 d B
\end{aligned}
$$

Moreover, as we can observe in (19) and (20), all amplitudes are function of $\sigma$, so one can set to zero and cancel the desired nonlinearity with an optimum design parameter for this purpose. Therefore, the model provides a wide range of possibilities of linearization including all design parameters of the modulator. Also, since the model starts from the effective index change versus DC bias voltage, any difference in dimensions or diode doping profile respect to the design values due to fabrication variations will change the curve and will be taken into account.

\section{B. Model results}

As it can be observed from the dependency of distortion amplitudes on the parameter $\sigma$ in the obtained analytical expressions, the phase shifter length is a parameter which strongly affects the distortion. So, there will be an optimum phase shifter length which allows linearizing the modulator cancelling the $3^{\text {rd }}$-order intermodulation product (dominant for less than one octave systems). For example, starting from a nonlinear effective index change curve as a function of DC bias voltage with $\mathrm{k}=0.52 .10^{-3}$ and $\mathrm{V}_{\mathrm{b}}=8 \mathrm{~V}$ in (3), in a quadrature bias modulator (modulator is designed such that the bias phase shift between the two arms at $\mathrm{V}_{\mathrm{DC}}=0$ and $\mathrm{V}_{\mathrm{RF}}=0$ is $\pi / 2$ ), one can linearize the modulator cancelling the $3^{\text {rd }}$-order nonlinearity knowing the optimum phase shifter length. Fig. 1 shows the lengths of the phase shifter which yield the most linear performance for several DC voltages. It can be observed, for example, that for a DC voltage of $1 \mathrm{~V}$ the modulator phase shifter length must be $825.5 \mu \mathrm{m}$.

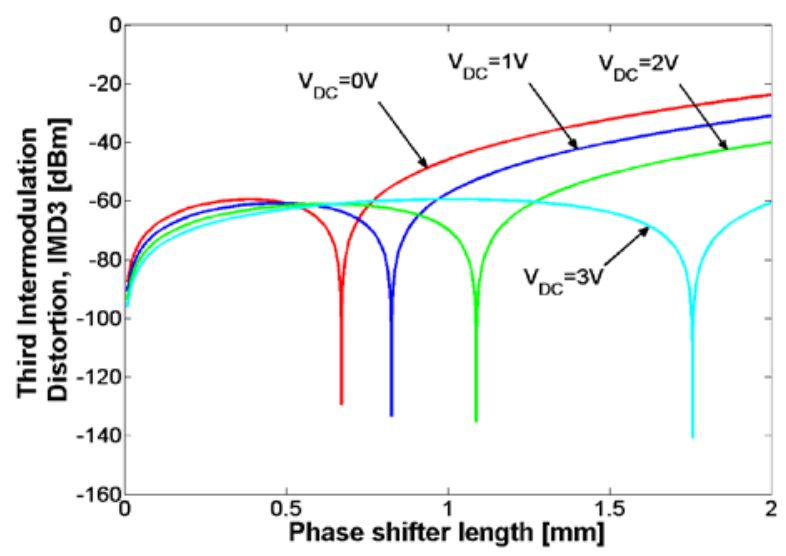

Fig. 1. Third-order intermodulation product power as a function of the phase shifter length in a $\Delta \Phi_{\text {Bias }}=\pi / 2$ modulator with a wavelength laser of $1550 \mathrm{~nm}$. 
Furthermore, as it can be deduced from (18), the nonlinearity of a silicon modulator also depends on the bias phase. Fig. 2 shows this dependency for a $1 \mathrm{~mm}$ MZI phase shifter length. This figure represents a contour of the $2^{\text {nd }}$-order (Fig. 2(a)) and $3^{\text {rd }}$-order (Fig. 2(b)) intermodulation distortions, $\mathrm{P}_{\mathrm{IMD} 2}$ and $\mathrm{P}_{\mathrm{IMD} 3}$ respectively, as a function of the DC voltage and bias phase. For example, for $1 \mathrm{~V}$ of DC we can eliminate the $3^{\text {rd }}$ order term if the bias phase is $0.47 \pi$. In such case, the $2^{\text {nd }}$ order intermodulation product would have a value of $-26.54 \mathrm{dBm}$. In the same way, for $4 \mathrm{~V}$ a bias phase shift of $0.15 \pi$ would result in the $3^{\text {rd }}$ order intermodulation term cancellation.

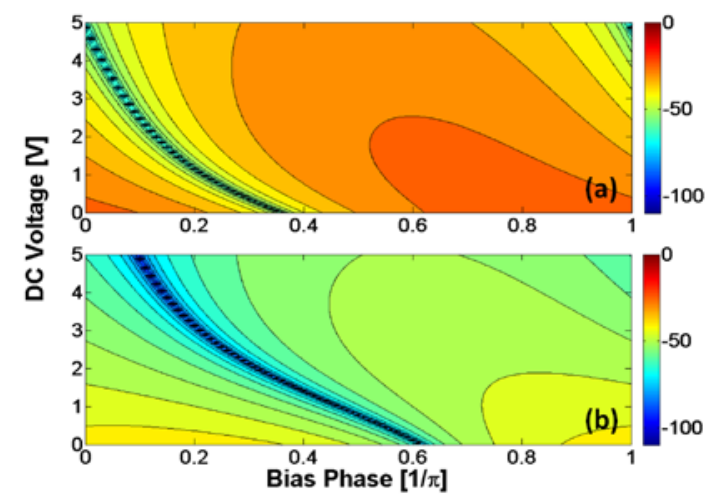

Fig. 2. Contour of $3^{\text {rd }}$ order (a) and $2^{\text {nd }}$ (b) intermodulation products power as a function of bias phase and DC bias voltage for a MZI with a phase shifter length of $1 \mathrm{~mm}$ and $\lambda=1550 \mathrm{~nm}$.

In order to corroborate our analytical model, we have performed a simulation in which the signal at the modulator output (time domain waveform) is converted to the frequency domain by performing a Fourier Fast Transfer (FFT). Then, one can select the desired frequency components and obtain its amplitude. This simulation allowed us to check the correct model performance since both simulation and analytic model show similar results. This can be observed in Fig. 3 where only some level error can be appreciated between simulation and our analytical model, but curves follow the same trend. In any case, the analytical expressions always overestimate the simulation results.

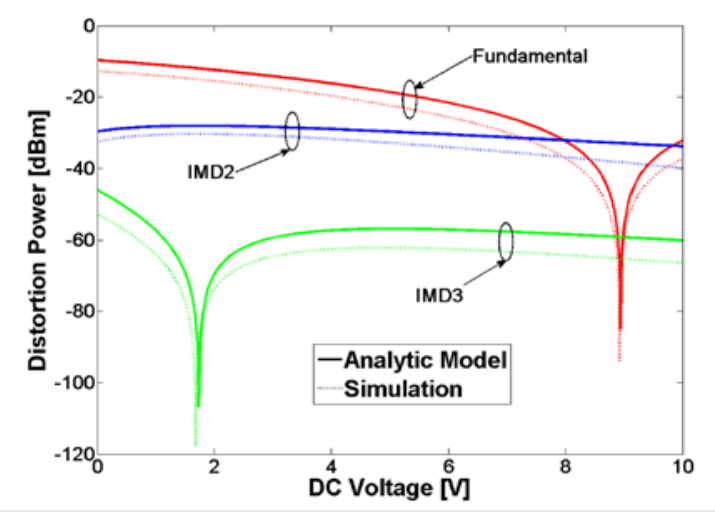

Fig. 3. Fundamental and intermodulation products power as a function of DC voltage in a fixed wavelength of $1550 \mathrm{~nm}$ for a $1 \mathrm{~mm}$ MZI under the condition $\Delta \Phi_{\text {Bias }}=\pi / 2$.

For a fixed quadrature modulator with an active length of $1 \mathrm{~mm}$ and a fixed 1550nm wavelength, we can obtain from Fig. 3 that approximately $1.7 \mathrm{~V}$ is the optimum DC voltage for cancelling the $3^{\text {rd }}$ order intermodulation term. Moreover we can observe in such Fig. 3 another point of view of the model application: for a DC voltage around $8.9 \mathrm{~V}$ the fundamental term vanishes and the $2^{\text {nd }}$ order intermodulation product is maximized. This point could be useful for a frequency upconversion process. The input signal can be set in the fundamental frequency and the signal information can be received in the $2^{\text {nd }}$ order term ensuring a good level of power in such signal.

It should be noticed that the free carrier concentration variation due to the plasma dispersion effect alters the imaginary part of the refractive index thus introducing absorption losses in the material. Such absorption losses usually depend on the applied voltage in a nonlinear form, which also needs to be taken into account in the nonlinear distortion analysis.

First, the effect of constant optical losses in the arms of the MZI on the nonlinear distortion has been analyzed by means of simulations. It has been obtained that having an unbalanced power distribution at the arms of the MZI only affects to the absolute value of the RF output power but the transmission response remains unaltered so, quality parameters as CDR or SFDR are not affected.

On the other hand, the dependency of absorption losses with voltage can also be approximated by a logarithmic form. Simulation results show that in this case the transmission response of the intermodulation products is red-shifted with respect to the response obtained with the developed model, i.e. the case of having no loss. Therefore, the variation of absorption losses $\left(\Delta \alpha=\alpha_{\max }-\alpha_{\min }\right)$ with voltage will reduce the accuracy of the model. However, the effect introduced by losses can be compensated in a real case by adjusting the bias $\left(\Phi_{\text {bias }}\right)$ for the target $\mathrm{V}_{\mathrm{DC}}$.

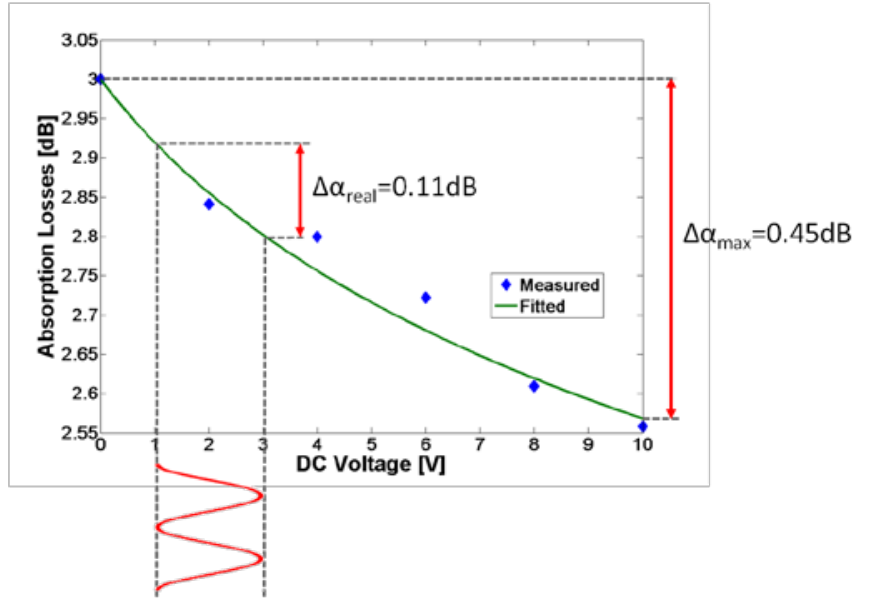

Fig. 4. Absorption losses variation with the DC voltage. The fitted curve is obtained from a logarithmic nonlinear approximation. It is shown both the maximum variation of absorption losses between $0 \mathrm{~V}$ and $10 \mathrm{~V}$ and the absorption losses variation with the voltage for $2 V_{\mathrm{pp}}$ of $\mathrm{RF}$ modulation voltage.

In the specific case of the $1 \mathrm{~mm}$ modulator under study in this work, the measured voltage-dependent absorption losses are very low so the effect is almost negligible. It has been obtained 
that the maximum variation is $\Delta \alpha=0.45 \mathrm{~dB}$ between 0 and $10 \mathrm{~V}$, that is, for $\Delta \mathrm{V}=10 \mathrm{~V}$. So, if the $\mathrm{RF}$ modulation voltage is around $2 \mathrm{~V}_{\mathrm{pp}}$, the variation of absorption losses with voltage will be of the order of 0.11dB, as shown in Fig. 4.
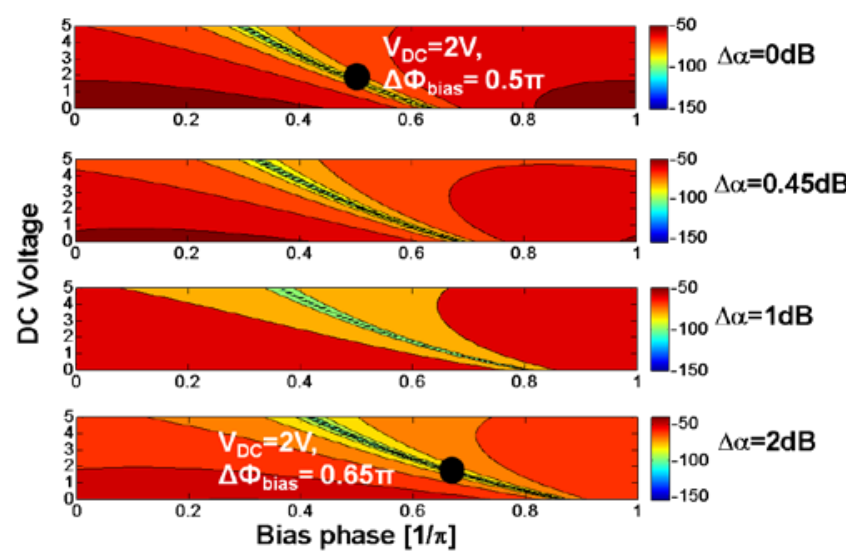

Fig. 5. RF output power of the IMD3 intermodulation product as a function of the bias phase and DC voltage for different values of absorption losses variation. The absorption losses compensation through $\Phi_{\text {bias }}$ parameter in a specific case for modulation linearization it is also shown.

Figure 5 shows the RF output power of the IMD3 intermodulation product as a function of the bias phase and DC voltage. It can be seen that the effect of losses are almost negligible for the case of $\Delta \alpha=0.45 \mathrm{~dB}$ but discrepancies are more noticeable as losses increases. However, if, for example, the IMD3 is minimized for $\mathrm{V}_{\mathrm{DC}}=2 \mathrm{~V}$ and a $\Phi_{\text {bias }}=0.5 \pi$. The effect of losses could be compensated just by slightly increasing $\Phi_{\text {bias }}$ to $0.65 \pi$ for a worst-case scenario of $\Delta \alpha=2 \mathrm{~dB}$. Therefore, the developed model remains as a powerful tool for analyzing nonlinear distortion in silicon MZI modulators.

\section{MODEL VALIDATION}

\section{A. Fabricated device structure}

The silicon-based modulator consists of an asymmetric MZI (as shown in Fig. 6) and the optical phase modulation is achieved by depleting the majority carriers from a reverse biased $P N$ junction located in the middle of the waveguide (Fig. 7(a)) and embedded in the shorter arm of the MZI.

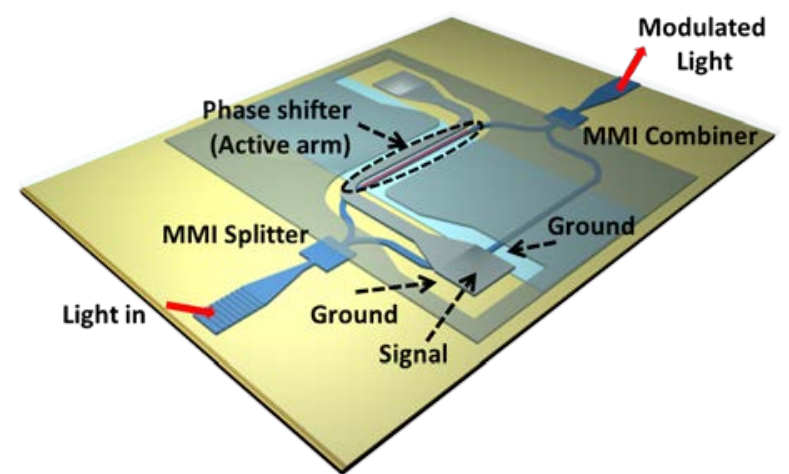

Fig. 6. Schematic of the fabricated silicon-based electro-optic MZI modulator. The MZI is asymmetric with a length difference of $180 \mu \mathrm{m}$ and the phase shifter in the shorter arm is $1 \mathrm{~mm}$ of length. MMI are used for divide the input light and combine the output modulated signal.
The length of the shorter arm ( $\left.\mathrm{L}_{\mathrm{ACT}}\right)$ of the MZI is $1 \mathrm{~mm}$ and the length difference between both arms $(\Delta \mathrm{L})$ is $180 \mu \mathrm{m}$ with MMI coupler splitter and combiner in the input and output respectively. The silicon waveguides of dimensions of $220 \mathrm{~nm}$ $\mathrm{x} 460 \mathrm{~nm}$ have been partially dry etched to leave a $100 \mathrm{~nm}$ thick slab. Doping concentrations in the p-and n-type regions of the diode are $4.10^{17} \mathrm{~cm}^{-3}$ and $5.10^{17} \mathrm{~cm}^{-3}$ respectively. The lumped electrode is formed by depositing a compound $\mathrm{AlCu}$ layer on top of the highly doped $\mathrm{p}^{+}$and $\mathrm{n}+$ regions with concentrations of $1.10^{20} \mathrm{~cm}^{-3}$ in order to obtain low resistive contacts. These are placed $450 \mathrm{~nm}$ and $500 \mathrm{~nm}$ for $\mathrm{p}^{+}$and $\mathrm{n}+$ respectively away from the rib edges to minimize the overlap between the optical mode and therefore reduce optical losses. As can be observed from Fig. 6, the incoming coherent light is coupled vertically to the chip via grating couplers. A traveling-wave groundsignal-ground (GSG) coplanar design has been used to enable further high-speed radio-frequency measurements (Fig. 7(b) and 7(c)). The complete fabrication process is described in [8].

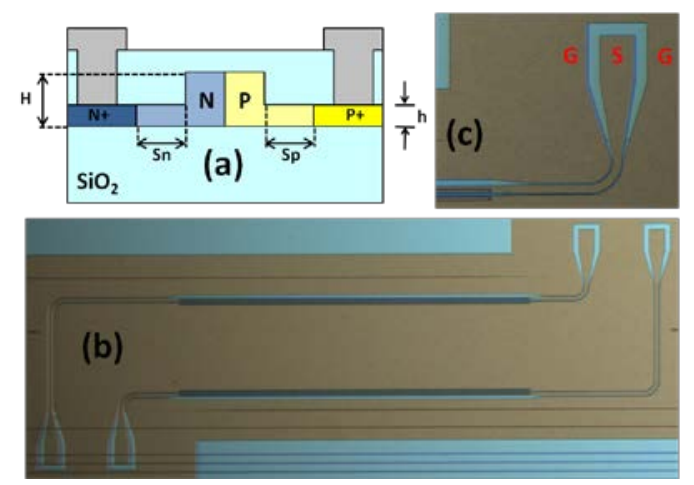

Fig. 7. (a) Schematic of the PN junction located at the middle of the active waveguide phase shifter, (b) Optical microscope image of the fabricated modulator with a clear view of the traveling-wave ground-signal-ground (GSG) coplanar, electrodes, (c) Detail of the GSG electrode.

As it was mentioned in the previous section, the model starts from the effective index change measurement, so, first of all, static and DC performance measurements have been carried out. 


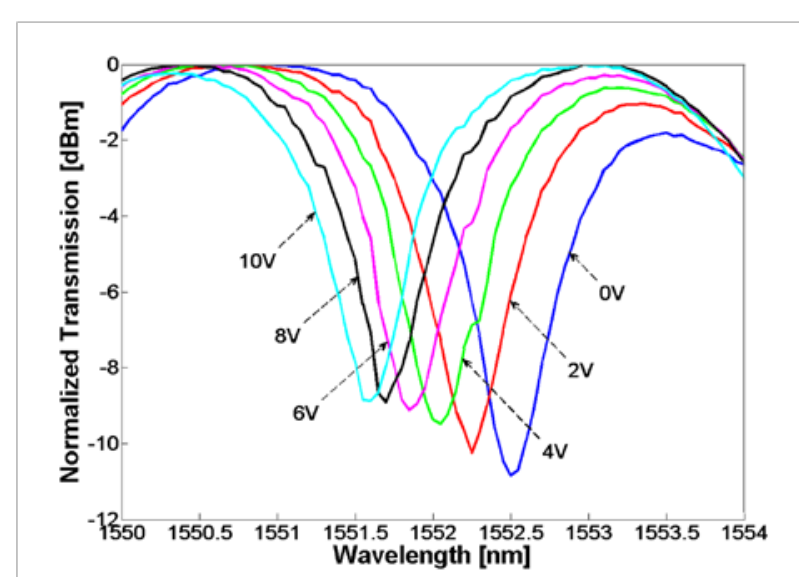

Fig. 8. Normalized DC transmission spectrum of the MZI modulator for different applied DC voltages.

The spectrum of the silicon-based MZI exhibits a free-spectral range (FSR) of $2.42 \mathrm{~nm}$ and a $10 \mathrm{~dB}$ drop in transmission at the resonant wavelength around 1552nm. Fig. 8 shows the MZI normalized transmission spectrum for varying DC voltages from $0 \mathrm{~V}$ to $10 \mathrm{~V}$.

As the modulator is asymmetric we have had to take into account this asymmetry in the bias phase as follows:

$$
\Delta \Phi_{\text {Bias }}=\Phi_{\text {Offset }}-\Delta \Phi_{\text {Asym. }}
$$

with:

$$
\Delta \Phi_{\text {Asym. }}=\frac{2 \pi}{\lambda} n_{g} \Delta L
$$

where $\mathrm{n}_{\mathrm{g}}$ is the group index and $\Delta \mathrm{L}$ is the length difference between both MZI arms. Moreover, the minus sign is because the active area is located in the shorter arm and thus the resonance shifts to lower wavelengths with the DC voltage. Finally, we have added an offset term ( $\left.\Phi_{\text {offset }}\right)$ which is required to fit the theoretical spectrum with the experimental one (in DC measurements). So, for a fixed arm difference, the bias point of the modulator can be tuned either through acting on the DC voltage or on the operation wavelength and viceversa. Taking into account (22) and (23) and plugging them into (18), one can obtain the optimum length difference and DC voltage for cancelling the desired intermodulation distortion. From the measured FSR, the group index has been calculated to be 3.9. On the other hand, the voltage-induced wavelength shift $(\Delta \lambda)$ in the MZI modulator output spectrum and the index group can be used to extract the effective index variations ( $\Delta$ neff), through (24):

$$
\Delta n_{\text {eff }}=\frac{\Delta \lambda}{\lambda} n_{g} \frac{\Delta L}{L_{A C T}}
$$

Figure 9 depicts the calculated and fitted effective index variation as a function of applied DC voltage. The fitted curve has been obtained from the nonlinear approximation described in (3).

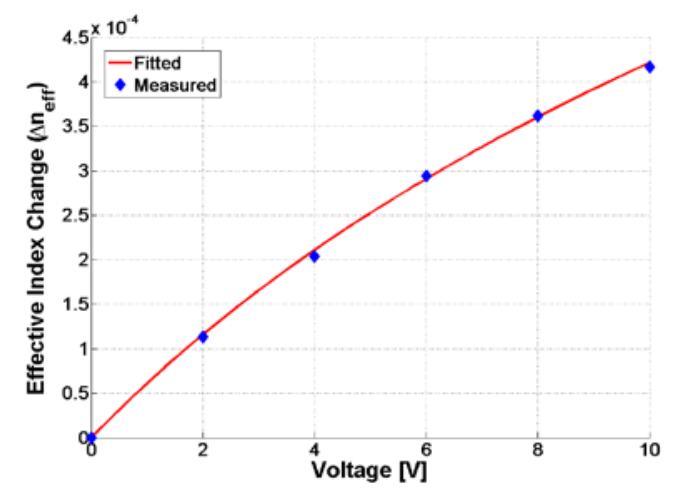

Fig. 9. Effective index change with the voltage. The measured data are obtained from the measured voltage-induced wavelength shift through equation (24). The fitted curve is obtained from nonlinear approximation described in (3).

\section{B. Experimental validation}

To validate the analytical model with experimental measurements we have performed a single-tone test and analyzed the harmonic distortion. The experimental set-up used for the RF analysis of the silicon-based electro-optic MZI modulator is shown in Fig. 10. The input light emitted by an External Cavity Laser (ECL) is coupled from standard single mode fibers to the chip via grating couplers. The polarization is optimized via the use of a polarization controller. One signal generator operating at $\mathrm{f}_{\mathrm{RF}}$ is coupled to a DC voltage using a bias-Tee and applied through high speed GSG probes to one port of the silicon MZI modulator while the other port is terminated with a $50 \Omega$ load. The output modulated signal is amplified with an erbium-doped fiber amplifier (EDFA), filtered by a tunable optical filter (OF) and split with a 50/50 optical splitter with the aim of photo-detecting and measuring the optical output by an optical power meter simultaneously. Finally, an electrical hybrid is used to separate the photodetected signal to a vectorial signal analyzer for measuring the electrical received signal in terms of frequency and to a digital communication analyzer for measuring the temporal signals.

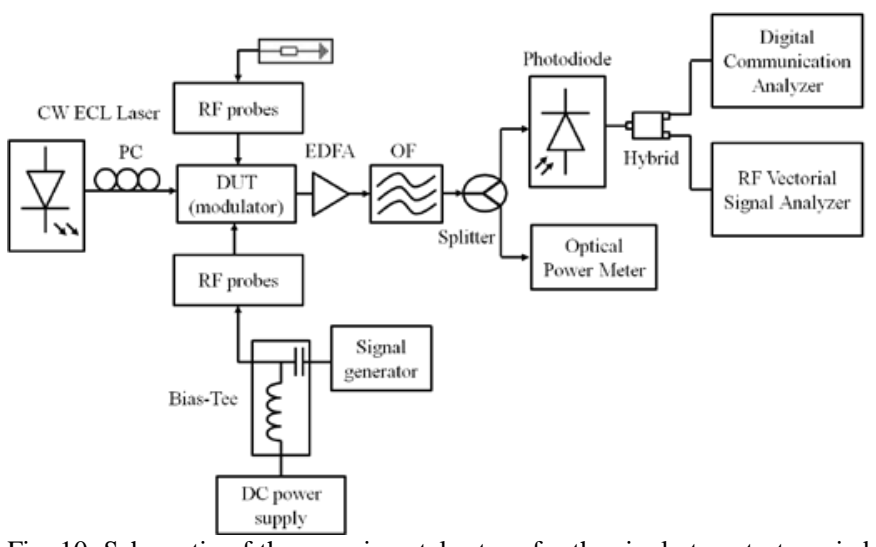

Fig. 10. Schematic of the experimental set-up for the single-tone test carried out to experimental validation of the model. 
As mentioned previously, due to the asymmetric MZI structure and the material in the active area, the bias point of the modulator can be tuned either through acting on the DC voltage or on the operation wavelength in contrast with conventional $\mathrm{LiNbO}_{3}$ modulators in which the bias point is driven only with a DC voltage. So, for a given wavelength, linear or nonlinear optimum operation can be achieved by adjusting the DC voltage and vice-versa. Thereby, representations of the distortion as a function of DC voltage and wavelength can help the designer to obtain the optimum combination wavelength-DC voltage point. Fig. 11 shows a contour of fundamental (P Pundam.) in Fig. 11(a), $2^{\text {nd }}$ harmonic $\left(\mathrm{P}_{\mathrm{HD} 2}\right)$ in Fig. 11(b) and $3^{\text {rd }}$ harmonic $\left(\mathrm{P}_{\mathrm{HD} 3}\right)$ in Fig. 11(c), obtained from analytical expressions for our fabricated device. So, Fig. 11 shows that for $1 \mathrm{~V}$ of $\mathrm{V}_{\mathrm{DC}}$ the HD2 is minimized at around $1551.25 \mathrm{~nm}$ and $\mathrm{HD} 3$ at $1551.75 \mathrm{~nm}$

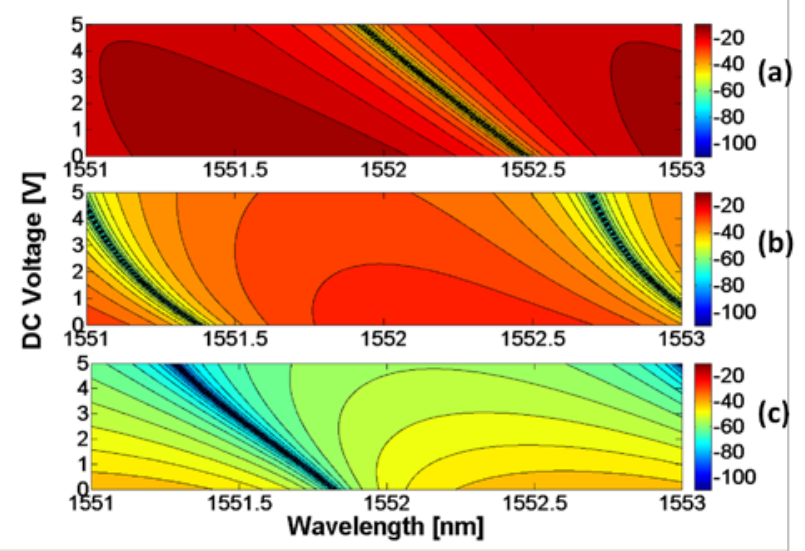

Fig. 11. Contour of the fundamental (a), the $2^{\text {nd }}(\mathrm{b})$ and the $3^{\text {rd }}$ order (c) harmonic products powers as a function of DC voltage and wavelength for our fabricated MZI.

- To validate these results, we first tested the model by measuring the harmonic distortion utilizing a fixed DC voltage of $1 \mathrm{~V}$ (Fig. 12) and tuning the operating wavelength. As it can be observed in the figure below, the measurements show the same trends and are in very good agreement with the analytic model corroborating the results obtained from Fig. 11. A slight displacement of $0.2 \mathrm{~nm}$ is produced in the HD3 term which is attributed to the characterization process. Moreover, it must be highlighted that the experimental curves are affected by the band-pass of the optical filter shape.

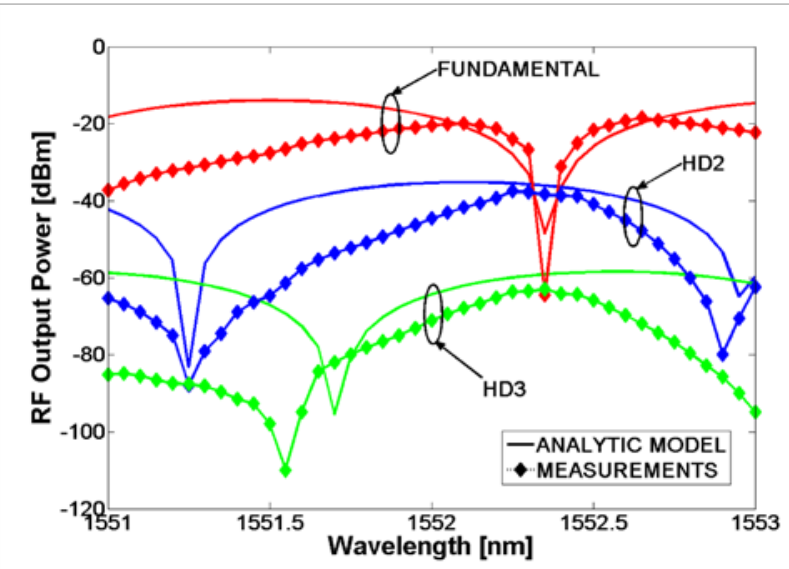

Fig. 12. Measured values and obtained curves from the analytical expressions of the fundamental and harmonic distortion performance as a function of the wavelength for a DC bias voltage of $1 \mathrm{~V}$. The RF input power of the single-tone is $10 \mathrm{dBm}\left(2 \mathrm{~V}_{\mathrm{pp}}\right)$.

The level of distortion using the carrier-to-distortion parameter can also be evaluated. Defining it as the ratio between the harmonic distortion product power and the received power fundamental tone:

$$
\begin{aligned}
& C D R_{2}=\frac{P_{H D 2}}{P_{\text {FUNDAM. }}} \\
& C D R_{3}=\frac{P_{\text {HD3 }}}{P_{\text {FUNDAM }}}
\end{aligned}
$$

Fig. 13 shows the experimental CDR (Fig. 13(a)), theoretical $\mathrm{CDR}_{2}$ (Fig. 13(b)) and $\mathrm{CDR}_{3}$ (Fig. 13(c)) as a function of the wavelength for $1 \mathrm{~V}$ of DC voltage. From that figure we can see that according to the analytical expressions, for $1 \mathrm{~V}$ maximum $\mathrm{CDR}$, around $20 \mathrm{~dB}$ for $\mathrm{CDR}_{2}$ and around $1 \mathrm{~dB}$ for $\mathrm{CDR}_{3}$, appears at $1552.4 \mathrm{~nm}$ as expected from Fig. 10 in which the fundamental is minimized. In contrast, minimum CDR, around $-56 \mathrm{~dB}$ for $\mathrm{CDR}_{2}$ and $-83.5 \mathrm{~dB}$ for $\mathrm{CDR}_{3}$ (obviously, the slight error displacement in HD3 appears again) are obtained at $1551.25 \mathrm{~nm}$ where HD2 is reduced and at $1551.55 \mathrm{~nm}$ where HD3 is reduced, respectively. So, we can affirm that the analytically estimated CDR is found to be in good agreement with the experimental one. 


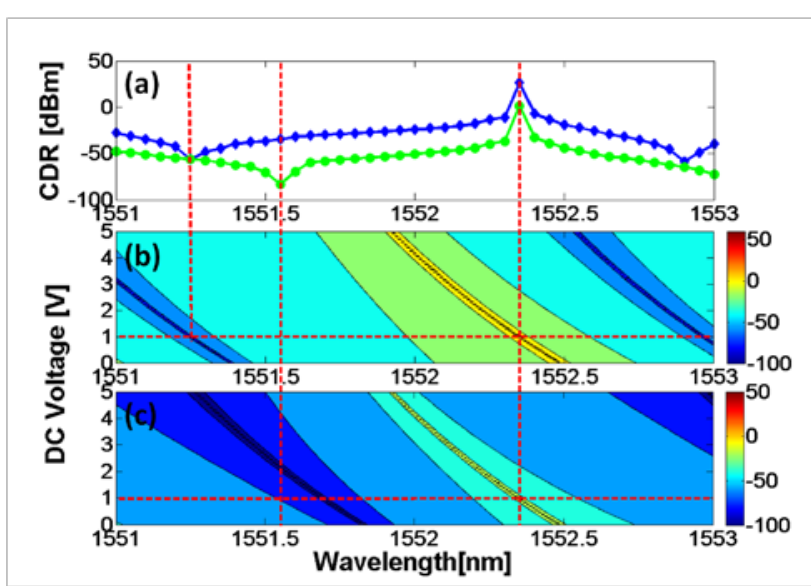

Fig. 13. Carrier-to-distortion ratio (a) from the experimental measurements and (b) CDR2 and (c) CDR3 from the analytical model. The RF input power of the single-tone is $10 \mathrm{dBm}\left(2 \mathrm{~V}_{\mathrm{nn}}\right)$.

Another type of measurement consists of the electrical response of the harmonic distortion as a function of the RF input power and fixing the operating wavelength and DC voltage. These results are shown in Fig. 12 for $1 \mathrm{~V}$ of DC bias at $\lambda=1552.1 \mathrm{~nm}$ (Fig. 14(a)) which is the wavelength where the measured fundamental signal is maximum and $\lambda=1552.4 \mathrm{~nm}$ (Fig. 14(b)) where it is minimum. As it can be observed, the fitted curves obtained from the analytical model fit with the experimental data. For example, in $1552.4 \mathrm{~nm}$ for an input RF power of $10 \mathrm{dBm}$, the fundamental tone remains under HD2 more than $20 \mathrm{~dB}$ and at around the same level power of HD3 as expected from Fig. 12 and Fig. 13.

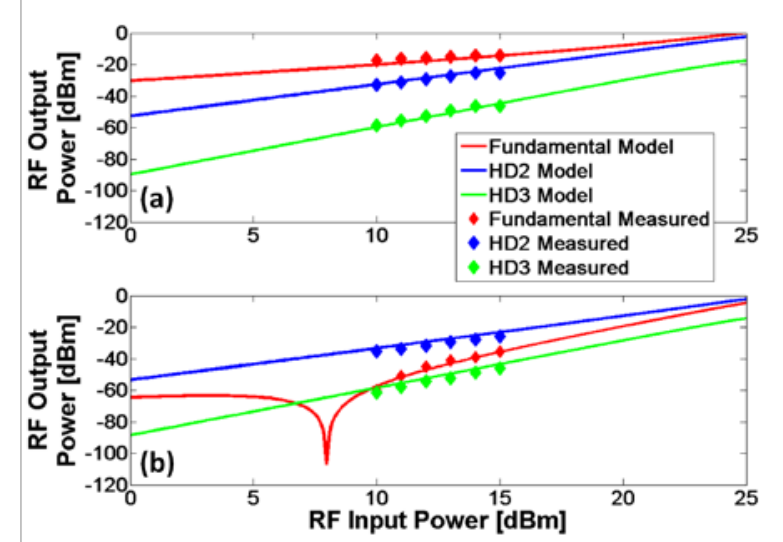

Fig. 14. Fundamental, HD2 and HD3 powers as a function of the modulating RF input power for (a) $\lambda=1552.1 \mathrm{~nm}$ and (b) $\lambda=1552.4 \mathrm{~nm}$. Curves are obtained from the analytical model and $\mathrm{VDC}_{\mathrm{DC}}=1 \mathrm{~V}$.

Finally, the modulation depth has been measured. It is well known that over-modulating a modulation device, which occurs when the RF modulating signal swing is too high, produces high distortion levels which strongly affect the signal output creating undesired harmonics. So, the modulation depth as a function of the RF input power has been obtained through the output temporal signals to corroborate that the measured harmonics in our fabricated device are only due to the modulator nonlinear feature and not due to over-modulation. This can be observed in Fig. 15 for $\lambda=1552.1 \mathrm{~nm}$. No modulation depth higher than the unit is obtained in the whole range of RF input powers measured. Figure inset shows an example of the measured temporal signal. In particular it corresponds to the case of $12 \mathrm{dBm}$ of $\mathrm{RF}$ input power which results in a modulation depth of around $50 \%$.

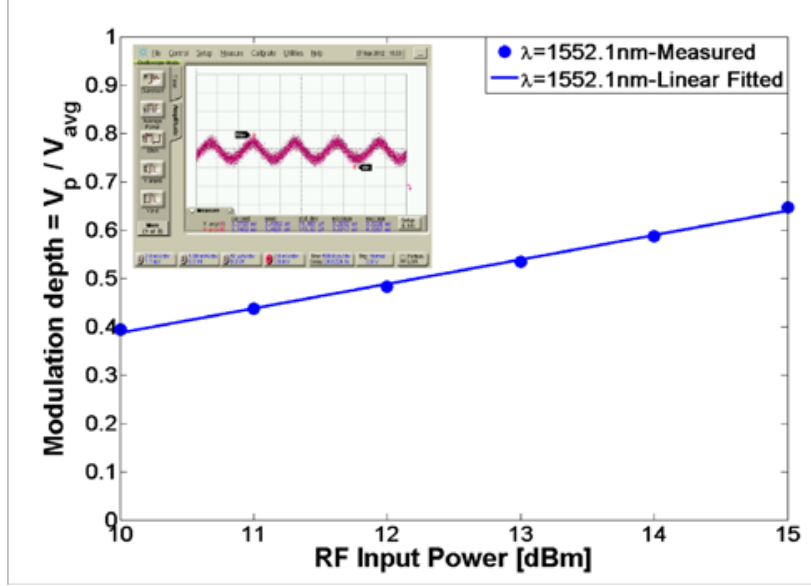

Fig. 15. Modulation depth as a function of the RF input power for the case of $\lambda=1552.1 \mathrm{~nm}$ and $\mathrm{V}_{\mathrm{DC}}=1 \mathrm{~V}$. It is observed that no over-modulation is produced.

\section{CONCLUSION}

We have developed analytical expressions for the harmonic and intermodulation nonlinear distortions of a silicon-based electro-optic Mach-Zehnder Interferometer modulator due to the nonlinear feature of the silicon and the modulator transfer function. Distortion equations are estimated analytically and found to be in good agreement with numerical simulations. Some of the model applications have been shown as obtaining the optimal phase shifter length or length difference between the MZI arms for cancelling the $3^{\text {rd }}$-order intermodulation distortion, allowing a linearized modulator. Finally, the harmonic distortion calculated by the analytical model has been validated by experimental results including the carrier-todistortion parameter showing excellent matching between the model results and experimental data. Given the growing advantages of silicon photonics and therefore of silicon modulators for emerging RoF applications, this model gives a potential tool to predict the nonlinear modulator performance and the parameters needed for a full study of an analog photonic link.

\section{ACKNOWLEDGMENT}

Authors acknowledge funding by the European Commission under project HELIOS (pHotonics Electronics functional Integration on CMOS), FP7-224312. P.S. and J.M. also acknowledge funding from TEC2012-38540 LEOMIS, TEC2008-06333 SINADEC and PROMETEO-2010-087. F.Y.G, D.J.T. and G.T.R. are supported by funding received from the UK EPSRC funding body under the grant "UK Silicon Photonics”. 


\section{REFERENCES}

[1] J. Capmany and D. Novak, "Microwave photonics combines two worlds,” Nature Photonics, vol. 1, no. 6, pp. 319-330, Jun. 2007.

[2] A. Seeds and K. Williams, "Microwave Photonics," J. Lightwave Technol. Vol. 24, pp. 4628-4641, 2006.

[3] R. A. Soref, “The past, present, and future of silicon photonics," IEEE J. Sel. Top. Quantum Electron., vol. 12, no. 6, pp. 1678-1687, 2006.

[4] B. Jalali and S. Fathpour, "Silicon Photonics," Journal of Lightwave Technology, vol. 24, no. 12, pp. 4600-4615, 2006.

[5] B. G. Lee, A. Biberman, J. Chan, and K. Bergman, "High-Performance Modulators and Switches for Silicon Photonic Networks-on-Chip," IEEE J. Sel. Top. Quantum Electron., vol. 16, pp. 6-22, 2010.

[6] G. T. Reed, G. Mashanovich, F. Y. Gardes and D. J. Thomson, "Silicon optical modulators", Nat. Photon., vol. 4, no. 8, pp. 518-526, 2010.

[7] R. Soref and B. Bennett, "Electrooptical effects in silicon," IEEE J. Sel. Top. Quantum Electron, vol.23, no.1, pp. 123- 129, Jan 1987.

[8] A. Brimont, A. M. Gutierrez, M. Aamer, D. J. Thomson, F. Y. Gardes, J. Fedeli, G. T. Reed, J. Marti, P. Sanchis, "Slow-Light-Enhanced Silicon Optical Modulators Under Low-Drive-Voltage Operation," Photonics Journal, IEEE , vol.4, no.5, pp.1306-1315, 2012

[9] F. Gardes, D. Thomson, N. Emerson, and G. Reed, "40 Gb/s silicon photonics modulator for TE and TM polarisations," Opt. Express, vol. 19, pp. 11804-11814, 2011.

[10] D.J. Thomson, F.Y. Gardes, J.-M. Fedeli, S. Zlatanovic, Hu Youfang, B.P.P. Kuo, E. Myslivets, N. Alic, S. Radic, G.Z. Mashanovich, G.T. Reed, "50-Gb/s Silicon Optical Modulator," IEEE Photonics Technology Letters, vol. 24, no. 4, pp. 234-236, Feb. 2012.

[11] A. Brimont, D. J. Thomson, F. Y. Gardes, J-M. Fedeli, G. T. Reed, J. Marti, P. Sanchis, "Low drive voltage $10 \mathrm{~Gb} / \mathrm{s}$ and high contrast $40 \mathrm{~Gb} / \mathrm{s}$ silicon slow wave modulators," 2012 IEEE 9th International Conference on Group IV Photonics (GFP), pp.195-197, 29-31 Aug. 2012.

[12] W. Green, M. Rooks, L. Sekaric, and Y. Vlasov, "Ultra-compact, low RF power, $10 \mathrm{~Gb} / \mathrm{s}$ siliconMach-Zehnder modulator," Opt. Express, vol. 15, pp. 17106-17113, 2007.

[13] S. Akiyama, T. Baba, M. Imai, T. Akagawa, M. Takahashi, N. Hirayama, H. Takahashi, Y. Noguchi, H. Okayama, T. Horikawa, and T. Usuki, "12.5-Gb/s operation with $0.29-\mathrm{V} \bullet \mathrm{cm} \mathrm{V} \pi \mathrm{L} \pi$ using silicon Mach-Zehnder modulator based-on forward-biased pin diode," Opt. Express, vol. 20, pp. 2911-2923, 2012.

[14] L. Liao, A. Liu, J. Basak, H. Nguyen, M. Paniccia, D. Rubin, Y. Chetrit, R. Cohen, N. Izhaky, "40 Gbit/s silicon optical modulator for highspeed applications," Electronics Letters, vol.43, no. 22, 2007.

[15] M. Ziebell, D. Marris-Morini, G. Rasigade, J. Fédéli, P. Crozat, E. Cassan, D. Bouville, and L. Vivien, "40 Gbit/s low-loss silicon optical modulator based on a pipin diode," Opt. Express, vol. 20, pp. 1059110596, 2012.

[16] J. Ding, H. Chen, L. Yang, L. Zhang, R. Ji, Y. Tian, W. Zhu, Y. Lu, P. Zhou, R. Min, and M. Yu, "Ultra-low-power carrier-depletion MachZehnder silicon optical modulator," Opt. Express, vol. 20, pp. 70817087, 2012.

[17] S. Manipatruni, X. Qianfan, B. Schmidt, J. Shakya, M. Lipson, "High Speed Carrier Injection $18 \mathrm{~Gb} / \mathrm{s}$ Silicon Micro-ring Electro-optic Modulator," Lasers and Electro-Optics Society, 2007. LEOS 2007, 20th Annual Meeting of the IEEE, pp.537-538, 2007.

[18] P. Dong, S. Liao, H. Liang, W. Qian, X. Wang, R. Shafiiha, D. Feng, G. Li, X. Zheng, A. Krishnamoorthy, and M. Asghari, "High-speed and compact silicon modulator based on a racetrack resonator with a $1 \mathrm{~V}$ drive voltage," Opt. Letters, vol. 35, pp. 3246-3248 (2010).

[19] G. Li, X. Zheng, J. Yao, H. Thacker, I. Shubin, Y. Luo, J. E. Cunningham, and A. V. Krishnamoorthy, "25Gb / s 1V-driving CMOS ring modulator with integrated thermal tuning,” Opt. Express, vol. 19 (21), pp. 20435-20443, 2011.

[20] A. Brimont, D. Thomson, F. Gardes, J. Fedeli, G. Reed, J. Martí, and P. Sanchis, "High-contrast $40 \mathrm{~Gb} / \mathrm{s}$ operation of a $500 \mu \mathrm{m}$ long silicon carrier-depletion slow wave modulator," Opt. Lett., vol. 37, pp. 35043506, 2012.

[21] W.B. Bridges, J.H. Schaffner, "Distortion in linearized electrooptic modulators," IEEE Transactions on Microwave Theory and Techniques, vol.43, no.9, pp.2184-2197, Sep 1995.
[22] A. Karim, J. Devenport, "Low Noise Figure Microwave Photonic Link," 2007 IEEE/MTT-S International Microwave Symposium, , vol., no., pp.1519-1522, 3-8 June 2007.

[23] G. Zhang, X. Zheng, S. Li, H. Zhang, and B. Zhou, "Postcompensation for nonlinearity of Mach-Zehnder modulator in radio-over-fiber system based on second-order optical sideband processing," Opt. Lett., vol. 37, pp. 806-808, 2012.

[24] A. Khilo, C. Sorace, and F. Kärtner, "Broadband linearized silicon modulator," Opt. Express, vol. 19, pp. 4485-4500, 2011.

[25] F. Vacondio, M. Mirshafiei, J. Basak, L. Ansheng, L. Ling, M. Paniccia, and L. A. Rusch, "A Silicon Modulator Enabling RF Over Fiber for 802.11 OFDM Signals," IEEE J. Sel. Top. Quantum Electron., vol. 16, pp. 141-148, 2010.

[26] S. Muping, Z. Lin, R. G. Beausoleil, and A. E. Willner, "Nonlinear Distortion in a Silicon Microring-Based Electro-Optic Modulator for Analog Optical Links," IEEE J. Sel. Top. Quantum Electron. vol. 16, pp. 185-191, 2010

[27] J. Marti, V. Polo, F. Ramos, and J. M. Fuster, "Single Mach-Zehnder Modulator Electro-Optical Harmonic Mixer for Broadband Microwave/Millimetre-Wave Applications," Wireless Personal Communications 15, 31-42 (2000).

[28] B. Cabon, "Microwave photonic mixing," Transactions on Computer Science \& Engineering and Electrical Engineering 17, 13 (2010).

[29] Gopalakrishnan, G.K.; Burns, W.K.; Bulmer, C.H.; , "Microwaveoptical mixing in LiNbO3 modulators," Microwave Theory and Techniques, IEEE Transactions on , vol.41, no.12, pp.2383-2391, Dec 1993

[30] Karim, A.; Devenport, J.; , "High Dynamic Range Microwave Photonic Links for RF Signal Transport and RF-IF Conversion," Lightwave Technology, Journal of , vol.26, no.15, pp.2718-2724, Aug.1, 2008

[31] V. Pagán, B. Haas, and T. Murphy, "Linearized electrooptic microwave downconversion using phase modulation and optical filtering," Opt. Express, vol. 19, pp. 883-895 (2011).

[32] A. Gutiérrez, A. Brimont, J. Herrera, M. Aamer, J. Martí, D. Thomson, F. Gardes, G. Reed, J. Fedeli, and P. Sanchis, "Silicon slow-light-based photonic mixer for microwave-frequency conversion applications," Opt. Lett., vol. 37, pp. 1721-1723, 2012.

[33] D. Marpaung, "High dynamic range analog photonic links design and implementation," University of Twente, The Netherlands, 2009.

[34] S. L. Chuang, "Physics of Optoelectronic devices," New York: Wiley, 1995.

A. M. Gutiérrez was born in Valencia, Spain in 1983. She received the Telecommunication Engineering degree from the Universitat Politecnica de Valencia as well as a Master Degree in technologies, systems and communications networks. She started a $\mathrm{PhD}$ in the Nanophotonics Technology Center in 2009. Her research interests include high speed silicon modulator modeling and characterization of ring resonators and slow wave structures.

Antoine Brimont was born in Valence, France, in 1982. He received the Engineering Master's Degree in Materials Science and Nanotechnology (M.Sc.) from the Institut National des Sciences Appliquées (INSA) de Rennes, France, as well as the Diplôme d'Études Approfondies (DEA) in Physics from the Université de Rennes 1, France, in 2005. He also received the Diploma de Estudios Avanzados (DEA) as well as the Ph.D. in Telecommunications Engineering from the Universitat Politècnica de València, Spain, in 2008 and 2011, respectively. He is currently working as a post-doctoral researcher at the Valencia Nanophotonics Technology Center, Spain. His research interests include active and passive silicon photonic devices and specifically high speed silicon modulators enhanced via slow light propagation. He has authored or co-authored over 35 papers in peerreviewed international journals and international conferences.

Javier Herrera was Telecommunication Engineer at 2000 and Telecommunication Engineer Ph. D. at 2005 by Universitat Politècnica de València. In 2000 he joined the Nanophotonics Technology Center at this university as junior research engineer. In 2006 he joined the COBRA Research Institute at Eindhoven University of Technology as senior research engineer, returning to Nanophotonics Technology Center at 2008 as senior research engineer. He has been involved in several research projects funded by the European Comission related to fiber-optics, photonics and broadband 
wireless systems and technologies such as IST-OBANET, ICT-LASAGNE, ICT-GANDALF, ICT-EUROFOS and ICT-FIVER. He has co-authored more than 80 journal and conference papers related to these technologies. In 2010 he joined FIBERNOVA SYSTEMS S. L. as R\&D engineer.

M. Aamer was born in Tangier, Morocco in 1982. She received the Telecommunication Engineering degree from the Universitat Politecnica de Valencia as well as a Master Degree in technologies, systems and communications networks. She started a $\mathrm{PhD}$ in the Nanophotonics Technology Center in 2009. Her research interests include silicon photonic devices as rotator and splitter as well as development of transceiver for acces networks in silicon photonics.

D. J. Thomson is a senior research fellow in the Optoelectronics Research Centre (ORC) at the University of Southampton. He started his silicon photonics research in 2004 as a PhD student at the University of Surrey under the guidance of Prof. Graham Reed. His PhD project involved investigating silicon based total internal reflection optical switches and more specifically methods of restricting free carrier diffusion within such devices. In 2008 he took up a role as a research fellow in the same research group leading the work package on silicon optical modulators within the HELIOS project. His research interests are optical modulation, optical switching, integration and packaging in silicon photonics. In 2011 David presented invited talks at SPIE Photonics West and IEEE Group IV Photonics conferences and in 2012 was selected to present his work at the SET for Britain event in the Houses of Parliament. In 2012 David was recruited to the University of Southampton. David has published over 75 papers/patents since 2006.

F. Y. Gardes is an Academic Fellow appointed to manage the optical modulation/detection and integration of the UK Silicon Photonics EPSRC programme. In 2005 Frederic initiated work on silicon optical depletion modulators and was the first to predict operation above 40GHz. In 2011 Frederic and his collaborators demonstrated optical modulation of up 50Gb/s with a quadrature ER of up to $10 \mathrm{~dB}$ at $40 \mathrm{~Gb} / \mathrm{s}$ setting a new state of the art performance for these devices. He is currently working with several national and international collaborators and is also involved in Silicon/Germanium QCSE devices, Germanium and defect induced detectors in silicon and active devices integration in group IV materials. Frederic Gardes is author of more than 65 publications, 2 patents and 5 book chapters in the field of Silicon Photonics. He is also a member of the programme committee of the IEEE Group IV Photonics conference.

Graham Reed is Professor of Silicon Photonics and Group Leader. He has recently joined Southampton from the University of Surrey, where he was Professor of Optoelectronics, and was Head of the Department of Electronic Engineering from 2006 to 2012. Reed is a pioneer in the field of Silicon Photonics, and acknowledged as the individual who initiated the research field in the UK. He established the Silicon Photonics Research Group at Surrey in 1989. The first Silicon Photonics company in the world, Bookham Technology Inc., was founded by Reed's PhD student, Dr Andrew Rickman, and adopted the research developed in the Group. The Silicon Photonics Group have provided a series of world leading results since it's inception, and are particularly well known for their work on silicon optical modulators. Reed is a regular invited and contributing author to the major Silicon Photonics conferences around the world. He has served on numerous international conference committees, and has also chaired many others. To name but two, he has been co-chair, of the Silicon Photonics symposium at Photonics West since it was first established in 2006, and in 2011 he was co-chair of the prestigious Silicon Photonics conference, IEEE Group IV Photonics, held at the Royal Society in London. He is currently a member of 5 international conference committees, and has published over 250 papers in the field of Silicon Photonics. He will co-chair 4 international symposia/conferences in 2012.

Jean-Marc Fédéli received the Electronics Engineer diploma from INPG Grenoble, France, in 1978. He conducted research with CEA-LETI on various magnetic memories and magnetic components as Project Leader, Group Leader, and Program Manager. For two years, he was Advanced Program Director with Memscap company for the development of RF-MEMS. He returned to CEA-LETI in 2002 as acted as coordinator of silicon photonic projects till 2012. Under a large partnership with universities and research laboratories, he worked on various technological aspects on photonics on CMOS (Si rib and stripe waveguides, Si3N4, and a-Si waveguides), Si modulators, Ge photodetectors, SiOx material, and InP sources on Si. He has been participating in a number of European FP6 projects (PICMOS, PHOLOGIC, MNTE, ePIXnet). Under European FP7, he was involved in the WADIMOS, PhotonFAB (ePIXfab)nad HELIOS projects. He is now managing the European project PLAT4M.

Pablo Sanchis received the Ingeniero de Telecomunicación degree (M.Sc.) and the Doctor Ingeniero de Telecomunicación degree (Ph.D.) from the Universidad Politécnica de Valencia, Valencia, Spain, in 2001 and 2005 respectively. He is full-time associate professor at the Universidad Politécnica de Valencia and senior researcher member of the Valencia Nanophotonics Technology Centre where he currently leads a research group in silicon active devices. His research interests include modelling, design and fabrication issues in integrated optics, especially in the field of silicon photonics. He has been involved in several national research projects and European research projects (IST-2000-25390 OBANET, IST-017158-PHOLOGIC, NoE-004525 ePIXnet, IST-224312 HELIOS). He has authored more than 45 papers in peer-reviewed international journals and more than 85 papers in international conferences and he holds 4 patents. 